\title{
Hydrogen isotope imaging in a compound Ca-Al-rich inclusion: insights on the origin of volatiles in the earliest solar system
}

\author{
JÉRÔME ALÉON ${ }^{1}$, DAN LEVY ${ }^{1,2,3}$, ALICE ALÉON- \\ TOPPANI $^{2}$, HÉLÈNE BUREAU ${ }^{4}$ AND HICHAM KHODJA ${ }^{5}$ \\ ${ }^{1}$ IMPMC, Museum National d'Histoire Naturelle, CNRS, \\ Sorbonne Université, IRD \\ ${ }^{2}$ IAS, Université Paris-Saclay, CNRS \\ ${ }^{3}$ IPRA, Université de Pau et des Pays de l'Adour \\ ${ }^{4}$ IMPMC - CNRS \\ ${ }^{5} \mathrm{NIMBE} / \mathrm{LEEL}$ \\ Presenting Author: jerome.aleon@mnhn.fr
}

Meteoritic Calcium, Aluminum-rich Inclusions (CAIs) are the oldest rocks formed in the solar system and are widely used as benchmarks for the initial composition of solar system materials. Because they are made of nominally anhydrous minerals (NAMs) formed at high temperature and very low pressure, their hydrogen isotopic composition is analytically challenging to measure. In order to gain insights on the origin of water in the solar system, we developed a novel approach for the analysis of $\mathrm{H}$ isotopes in NAMs using $\mathrm{H}$ isotopic imaging by NanoSIMS on thin sections prepared by focused ion beam (Lévy et al. 2019a) and studied the $\mathrm{H}$ isotopic composition of E101.1, a compound CAI from the Efremovka reduced CV3 chondrite, where secondary minerals are thought to have a pre-accretionary origin (Lévy et al. 2019b). Primary minerals have extremely low D/H ratios, with $\delta \mathrm{D}$ values down to $-850 \%$, recording the trapping of solar/nebular hydrogen. Secondary FeO-rich minerals from a xenolithic inclusion have $\delta \mathrm{D}$ values up to $+1000 \%$, which is attributed to diffusive $\mathrm{H}$ loss during capture of the fragments by the partially molten host. Hence they formed before capture with elevated water content, in a nebular environment where the oxygen fugacity was significantly higher than that of the solar gas. The $\mathrm{H}$ isotopic composition of this oxidizing reservoir was within $20 \%$ of that of the terrestrial oceans. $\mathrm{H}$ isotopes further correlate with oxygen and nitrogen isotopes. This indicates that inner solar system volatiles including water already had a planetary isotopic composition when CAI formed, i.e. during collapse of the protosolar cloud core, within the first $2 \times 10^{5}$ years of the solar system.

References:

Lévy, Aléon, Aléon-Toppani, Troadec, Duhamel, GonzalezCano, Bureau, Khodja (2019a), Analytical Chemistry 91, 1376313771.

Lévy, Aléon, Aléon-Toppani, Brunetto (2019b) 82nd Annual Meeting of The Meteoritical Society, LPI Contribution No. 2157, 2019, id.6046 